\title{
OPTIMAL STOCHASTIC CONTROL OF MEASURE SOLUTIONS ON HILBERT SPACE
}

\author{
N.U. Ahmed ${ }^{1}$ \\ ${ }^{1}$ University of Ottawa, SITE \& Department of Mathematics, ahmed@ site.uottawa.ca
}

\begin{abstract}
This paper is concerned with optimal control of semilinear stochastic evolution equations on Hilbert space driven by stochastic vector measure. Both continuous and discontinuous (measurable) vector fields are admitted. Due to nonexistence of regular solutions, existence and uniqueness of generalized (or measure valued) solutions are proved. Using these results, existence of optimal feedback controls from the class of bounded Borel measurable maps are proved for several interesting optimization problems.
\end{abstract}

keywords: Stochastic Differential Equations, Hilbert Space, Measurable Vector Fields, Finitely Additive Measure Solutions, Optimal Feedback Controls.

\section{Introduction}

For motivation let us consider the deterministic evolution equation

$$
\dot{x}=A x+F(x), t \geq 0, x(0)=x_{0},
$$

in a Hilbert space $H$ where $A$ is the infinitesimal generator of a $C_{0}$-semigroup, $S(t), t \geq 0$, on $H$ and $F: H \longrightarrow H$ is a continuous map. It is well known that if $H$ is finite dimensional, mere continuity of $F$ is good enough to prove existence of local solutions with possibly finite blow up time. If $H$ is an infinite dimensional Hilbert space continuity no longer guarantees existence of even local solutions unless the semigroup $S(t), t>0$, is compact. Because of this, the very notion of solutions required a major generalization to cover continuous as well as discontinuous vector fields [11], [1],[2],[3],[4],[5]. Using the general concept of measure solutions one can completely avoid standard assumptions such as local Lipschitz property and linear growth for both the drift and the diffusion operators as often used in [8] and [11].

Many physical models are described by differential equations with discontinuous and possibly unbounded vector fields without satisfying any of the standard regularity properties including monotonicity or accretivity etc. In this

Please use the following format when citing this chapter:

Ahmed, N.U., 2006, in IFIP International Federation for Information Processing, Volume 202, Systems, Control, Modeling and Optimization, eds. Ceragioli, F., Dontchev, A., Furuta, H., Marti, K., Pandolfi, L., (Boston: Springer), pp. 1-12. 
situation there is no solution in the sense of any one of the standard notions (classical,strong,mild,weak). Even the weak solution, if one exists, is highly irregular. However under fairly general assumptions one is able to prove existence of measure valued solutions. Functionals (physical observables) of such measure solutions, such as

$$
\Upsilon(\mu) \equiv E \int_{I} f\left(\mu_{t}\left(\varphi_{1}\right), \mu_{t}\left(\varphi_{2}\right) \cdots, \mu_{t}\left(\varphi_{m}\right)\right) \rho(d t),
$$

where $f \in B C\left(R^{m}\right), \varphi_{i} \in B C(H)$ and $\rho$ is a countably additive bounded positive measure on $I$ having bounded total variation, are of practical interest. For example, in the study of hydrodynamic turbulence, the solution of Euler equation, as the limit of the Navier-Stokes equation with the Reynolds number increasing towards infinity, can be considered as a measure solution. Its impact on mixing is an important physical variable.

Let $\{H, \Xi, E\}$ be any three Hilbert spaces relating the stochastic system governed by an evolution equation of the form

$$
\begin{aligned}
d x(t)= & A x(t) d t+ \\
& \\
& +G(x(t)) d t+\Gamma(x(t)) u(t, x(t)) d t \\
x(0)= & x_{0} .
\end{aligned}
$$

Here $A$ and $F$ are as described above, and $G: H \longrightarrow \mathcal{L}(E, H)$ is a continuous map and $\Gamma: H \longrightarrow \mathcal{L}(\Xi, H)$ is Borel measurable map and $M$ is an $E$-valued stochastic vector measure defined on the sigma algebra $\mathcal{B}_{0}$ of Borel subsets of $R_{0} \equiv[0, \infty)$.

The last term on the right hand side of equation (2) gives rise to a well defined stochastic integral since the integrand is adapted and therefore previsible or equivalently measurable with respect to the sigma algebra of predictable sets. An intuitive physical meaning is as follows. Given that $\{t\}$ is an atom of the measure $M$, the state at time $t$ is uniquely determined by the state $x(t-)$ (just before the jump) and the jump size $M(\{t\})$ of the measure $M$.

For simplicity of presentation, we have considered the operators $F, G, \Gamma$ to be independent of time. However the results presented here can be extended to the time varying case without any difficulty.

The paper is organized as follows. In section 2 we recall some important facts from analysis sufficient to serve our needs. In section 3 and 4 , we consider system (2) and present without proof two results on existence of measure valued solutions and their regularity properties. Using these results, in section 5 , we consider control problems and present several results on the question of existence of optimal feedback controls. 


\section{Basic Facts from Analysis}

Recently the author dealt with the question of existence of measure valued solutions for semilinear stochastic differential equations with continuous but unbounded nonlinearities driven by cylindrical Brownian motion [3]. Here we admit Borel measurable, possibly unbounded, vector fields and replace the Brownian motion by a more general stochastic vector measure. Properties of the stochastic vector measure are stated in the sequel.

\section{Radon Nikodyme Property \& Lifting:}

For any normal topological space $Z$, let $B C(Z)$ and $B(Z)$ denote the vector spaces of bounded continuous and bounded Borel measurable functions on $Z$ respectively. Furnished with the supnorm topology these are Banach spaces. It follows from a well known result [10] that the corresponding duals are given by $\Sigma_{r b a}(Z)\left(\Sigma_{b a}(Z)\right)$ which are regular bounded (bounded) finitely additive measures on the algebra of sets determined closed subsets of $Z$. Note that the dual pairs $\left\{B C(Z), \Sigma_{r b a}(Z)\right\}$ and $\left\{B(Z), \Sigma_{b a}(Z)\right\}$ do not satisfy RadonNikodym (RNP) property [8]. Hence, for any finite measure space $(S, \mathcal{S}, \gamma)$, it follows from the theory of lifting that the dual of $L_{1}(S, B C(Z))$ is given by $L_{\infty}^{w}\left(S, \Sigma_{r b a}(Z)\right)$. These are weak star measurable measure valued functions. To study the question of existence, we need these spaces.

\section{Special Vector Spaces Used:}

Now we are prepared to introduce the vector spaces used in the paper. Let $H, E$ be two separable Hilbert spaces and $\left(\Omega, \mathcal{F}, \mathcal{F}_{t} \uparrow, t \geq 0, P\right)$ a complete filtered probability space, $M(J), J \in \mathcal{B}_{0}$, an $E$ valued $\mathcal{F}_{t}$ adapted vector measure in the sense that for any $J \in \mathcal{B}_{0}$ with $J \subset[0, t], M(J)$ or more precisely $e^{*}(M(J))$ is $\mathcal{F}_{t}$ measurable for every $e^{*} \in E^{*}=E$. For the purpose of this paper we consider $\mathcal{F}_{t} \equiv \mathcal{F}_{t}^{M} \vee \sigma\left(x_{0}\right)$, where $\mathcal{F}_{t}^{M}, \sigma\left(x_{0}\right)$ are the smallest sigma algebras with respect to which the measures $M$ and the initial state $x_{0}$ respectively are measurable. Let $I \times \Omega$ be furnished with the predictable $\sigma$-field with reference to the filtration $\mathcal{F}_{t}, t \in I$ and $M_{\infty, 2}^{w}\left(I \times \Omega, \Sigma_{r b a}(H)\right) \subset L_{\infty, 2}^{w}\left(I \times \Omega, \Sigma_{r b a}(H)\right)$ denote the vector space of $\Sigma_{r b a}(H)$ valued random processes $\left\{\lambda_{t}, t \in I\right\}$, which are $\mathcal{F}_{t}$-adapted and $w^{*}$-measurable in the sense that, for each $\phi \in B C(H)$, $t \longrightarrow \lambda_{t}(\phi)$ is a bounded $\mathcal{F}_{t}$ measurable random variable possessing finite second moments. We furnish this space with the $w^{*}$ topology. Clearly this is the dual of the Banach space

$$
\left.M_{1,2}(I \times \Omega, B C(H)) \subset L_{1,2}(I \times \Omega, B C(H))\right),
$$

where the later space is furnished with the natural topology induced by the norm given by

$$
\|\varphi\| \equiv \int_{I}\left(\mathcal{E}(\sup \{|\varphi(t, \omega, \xi)|, \xi \in H\})^{2}\right)^{1 / 2} d t .
$$


Similarly, one can verify that $M_{\infty, 2}^{w}\left(I \times \Omega, \Sigma_{b a}(H)\right)$ is the dual of the Banach space $M_{1,2}(I \times \Omega, B(H))$. We will have occasion to use both these spaces.

\section{Basic properties of $M$ :}

(M1): $\left\{M(J), M(K), J \cap K=\emptyset, J, K \in \mathcal{B}_{0}\right\}$ are pair wise independent $E$ valued random variables (vector measures) satisfying $\mathcal{E}\{(M(J), \xi)\}=0, J \in$ $\mathcal{B}_{0}, \xi \in E$, where $\mathcal{E}(z) \equiv \int_{\Omega} z P(d \omega)$.

(M2): There exists a countably additive bounded positive measure $\pi \in$ $M_{c}\left(R_{0}\right)$, having bounded total variation on bounded sets, such that for every $\xi, \zeta \in E$,

$$
\mathcal{E}\{(M(J), \xi)(M(K), \zeta)\}=(\xi, \zeta)_{E} \pi(J \cap K) .
$$

Clearly, it follows from this last property that, for any $\xi \in E, \mathcal{E}\left\{(M(J), \xi)^{2}\right\}=$ $|\xi|_{E}^{2} \pi(J)$, and that the process $N$, defined by

$$
N(t) \equiv \int_{0}^{t} M(d s), t \geq 0,
$$

is a square integrable $E$-valued $\mathcal{F}_{t}$-martingale. Gyongi and Krylov [12] have also considered general noise for a special class of finite dimensional systems.

\section{EXISTENCE OF MEASURE SOLUTIONS}

In recent years a notion of generalized solution, which consists of regular finitely additive measure valued functions, has been extensively used in the study of semi linear and quasi linear systems with vector fields which are merely continuous and bounded on bounded sets; see [1],[2],[3], [11] and the references therein. Existence of solutions for deterministic systems, such as (1), was proved in $[1,2,11]$ with varying degrees of generality. Recently [6] existence of measure solutions for stochastic system (2), generalizing a previous result of the author [3], has been proved. These latest results cover Borel measurable drift and diffusion assumed to be merely bounded on bounded sets. Our main objective here is to prove existence of optimal feedback controls for these class of systems.

Since the measure solutions may not be fully supported on the original state space $H$, it is useful to extend the state space to a compact Hausdorff space containing $H$ as a dense subspace. Since every metric space is a Tychonoff space, $H$ is a Tychonoff space. Hence $H^{+} \equiv \beta H$, the Stone-Čech compactification of $H$, is a compact Hausdorff space and consequently bounded continuous functions on $\mathrm{H}$ can be extended to continuous functions on $\mathrm{H}^{+}$. In view of this we shall often use $H^{+}$in place of $H$ and the spaces $M_{1,2}\left(I \times \Omega, B C\left(H^{+}\right)\right)$with dual $M_{\infty, 2}^{w}\left(I \times \Omega, \Sigma_{r b a}\left(H^{+}\right)\right) \supset M_{\infty, 2}^{w}\left(I \times \Omega, \Pi_{r b a}\left(H^{+}\right)\right)$. Here $M_{\infty, 2}^{w}(I \times$ $\left.\Omega, \Pi_{r b a}\left(H^{+}\right)\right)$is the set of all finitely additive probability measure valued processes, a subset of the vector space $M_{\infty, 2}^{w}\left(I \times \Omega, \Sigma_{r b a}\left(H^{+}\right)\right)$. Note that, since 
$H^{+}$is a compact Hausdorff space, $\Sigma_{r b a}\left(H^{+}\right)=\Sigma_{r c a}\left(H^{+}\right)$. In view of the fact that the measure solutions of stochastic evolution equations restricted to $H$ are only finitely additive, we prefer to use the notation $\Sigma_{r b a}\left(H^{+}\right)$to emphasize this fact though they are countably additive on $\mathrm{H}^{+}$.

Without further notice, throughout this paper we use $D \phi$ and $D^{2} \phi$ to denote the first and second Frechet derivatives of the function $\phi$ whenever they exist. We denote by $\Psi$ the class of test functions as defined below:

$$
\Psi \equiv\left\{\phi \in B C(H): D \phi, D^{2} \phi \text { exist, continuous and bounded on } H\right\} .
$$

Define the operators $\mathcal{A B}$ and $\mathcal{C}$ with domains given by

$$
\begin{aligned}
& \mathcal{D}(\mathcal{A}) \equiv\left\{\phi \in \Psi: \mathcal{A} \phi \in B C\left(H^{+}\right)\right\} \\
& \mathcal{D}(\mathcal{B}) \equiv\left\{\phi \in \Psi: D \phi \in D\left(A^{*}\right) \& \mathcal{B} \phi \in B C\left(H^{+}\right)\right\},
\end{aligned}
$$

where

$$
\begin{aligned}
& (\mathcal{A} \phi)(\xi)=(1 / 2) \operatorname{Tr}\left(D^{2} \phi G G^{*}\right)(\xi), \phi \in \mathcal{D}(\mathcal{A}) \\
& \mathcal{B} \phi=\left(A^{*} D \phi(\xi), \xi\right)+(F(\xi), D \phi(\xi)) \text { for } \phi \in \mathcal{D}(\mathcal{B}) \\
& \mathcal{C} \phi(\xi) \equiv G^{*}(\xi) D \phi(\xi) .
\end{aligned}
$$

First we consider the uncontrolled system

$$
\begin{aligned}
& d x(t)=A x(t) d t+F(x(t)) d t+G(x(t-)) M(d t), \\
& x(0)=x_{0}
\end{aligned}
$$

and use the notion of measure (generalized) solutions introduced in [3] and finally add modifications necessary for the control system.

DEFINITION 1 Ameasure valued random process $\mu \in M_{\infty, 2}^{w}\left(I \times \Omega, \Pi_{r b a}\left(H^{+}\right)\right)$ is said to be a measure (or generalized) solution of equation (4) if for every $\phi \in \mathcal{D}(\mathcal{A}) \cap \mathcal{D}(\mathcal{B})$ and $t \in I$, the following equality holds

$$
\begin{aligned}
\mu_{t}(\phi)= & \phi\left(x_{0}\right)+\int_{0}^{t} \mu_{s}(\mathcal{A} \phi) \pi(d s)+\int_{0}^{t} \mu_{s}(\mathcal{B} \phi) d s \\
& +\int_{0}^{t}<\mu_{s-}(\mathcal{C} \phi), M(d s)>_{E} P-a . s .
\end{aligned}
$$

where $\mu_{t}(\psi) \equiv \int_{H^{+}} \psi(\xi) \mu_{t}(d \xi), t \in I$.

Again the stochastic integral is well defined since the integrand is predictable and the process $\mu_{t}, t \in I$, is cadlag in the weak star sense. This follows from the assumption that the compensating measure $\pi$ has bounded total variation and hence $M$ can have at most a countable set of atoms. 
REMARK 2 Note that equation (5) can be written in the differential form as follows:

$$
d \mu_{t}(\phi)=\mu_{t}(\mathcal{A} \phi) \pi(d t)+\mu_{t}(\mathcal{B} \phi) d t+<\mu_{t-}(\mathcal{C} \phi), M(d t)>
$$

with $\mu_{0}(\phi)=\phi\left(x_{0}\right)$. This is in fact the weak form of the stochastic evolution equation

$$
d \mu_{t}=\mathcal{A}^{*} \mu_{t} \pi(d t)+\mathcal{B}^{*} \mu_{t} d t+<\mathcal{C}^{*} \mu_{t-}, M(d t)>_{E}, \mu_{0}=\delta_{x_{0}},
$$

on the state space $\Sigma_{\text {rba }}(H)$ where $\left\{\mathcal{A}^{*}, \mathcal{B}^{*}, \mathcal{C}^{*}\right\}$ are the duals of the operators $\{\mathcal{A}, \mathcal{B}, \mathcal{C}\}$.

In case the system (2) has a weak solution in the classical sense, the measure solution degenerates into a Dirac measure concentrated along the path and the expression (5) defines the weak solution.

To proceed further we shall need the following

Assumption $3(A l)$ : there exists a sequence $\left\{F_{n}, G_{n}\right\}$ with $F_{n}(x) \in D(A)$ and $G_{n}(x) \in \mathcal{L}(E, D(A))$, for each $x \in H$, and

$$
F_{n}(x) \stackrel{\tau_{w u c}}{\longrightarrow} F(x) \text { in } H \text { and } G_{n}(x) \stackrel{\tau_{\text {souc }}}{\longrightarrow} G(x) \text { in } \mathcal{L}(E, H),
$$

where $\tau_{\text {wuc }}\left(\tau_{\text {souc }}\right)$ denotes the topology of weak convergence (convergence in strong operator topology) uniformly on compacts.

(A2): there exists a pair of sequence of real numbers $\left\{\alpha_{n}, \beta_{n}>0\right\}$, possibly $\alpha_{n}, \beta_{n} \rightarrow \infty$ as $n \rightarrow \infty$, so that both $F_{n}, G_{n}$ are Lipschitz having linear growth with coefficients $\alpha_{n}, \beta_{n}$ respectively.

We note that under the very relaxed assumptions used here, nonlinearities having polynomial growth are also admissible.

Following result generalizes our previous result ([3], Theorem 3.2).

THEOREM 4 Suppose $A$ is the infinitesimal generator of a $C_{0}$-semigroup in $H$ and the maps $F: H \longrightarrow H, G: H \longrightarrow \mathcal{L}(E, H)$ are continuous, and bounded on bounded subsets of $H$, satisfying the approximation properties (A1) and (A2); and $M$ is the vector measure satisfying (M1) and (M2). Then, for every $x_{0}$ for which $P\left\{\omega \in \Omega:\left|x_{0}\right|_{H}<\infty\right\}=1$, the evolution equation (4) has at least one measure valued solution

$$
\lambda^{0} \in M_{\infty, 2}^{w}\left(I \times \Omega, \Sigma_{r b a}\left(H^{+}\right)\right)
$$

in the sense of Definition 1. Further, $\lambda^{0} \in M_{\infty, 2}^{w}\left(I \times \Omega, \Pi_{\text {rba }}\left(H^{+}\right)\right)$.

Proof. Proof will appear in [6]. 
Supports of measure solutions may leak out to infinity in the sense that $\lambda_{t}^{0}(H)<1$ while $\lambda_{t}^{0}\left(H^{+}\right)=1$ with probability one. For any $\beta>0$ and $t \in I$, the operator $Q_{t}^{\beta}$ as given below is well defined

$$
\left(Q_{t}^{\beta} \xi, \xi\right) \equiv E \int_{H^{+}}(x, \xi)^{2} \exp -\left\{\beta|x|^{2}\right\} \lambda_{t}^{0}(d x)
$$

It follows from Milnos-Sazanov theorem that if $\lim _{\sup _{\beta \downarrow 0}} \operatorname{Tr}\left(Q_{t}^{\beta}\right)<\infty$ then we are assured of $H$ being the support of the measure solution. If $\lambda_{t}^{o}$ is not supported on $H$, we can still define an $H$-valued process given by

$$
X_{t}^{\beta} \equiv \int_{H^{+}} x \exp -\left\{\beta|x|_{H}^{2}\right\} \lambda_{t}^{0}(d x)
$$

which can serve as the approximate path process becoming highly irregular with decreasing $\beta$ and possibly escaping to $H^{+}$as $\beta \downarrow 0$.

REMARK 5 In view of Theorem 4, F, G are required to be merely continuous and bounded on bounded sets and hence they may have polynomial growth [3]. In contrast, for standard mild solutions it is usually assumed that F, G are locally Lipschitz having at most linear growth [8].

The following corollary is an immediate consequence of Theorem 4.

COROLLARY 6 Consider the forward Kolmogorov equation,

$$
d \vartheta_{t}=\mathcal{A}^{*} \vartheta_{t} \pi(d t)+\mathcal{B}^{*} \vartheta_{t} d t, \vartheta(0)=\nu_{0},
$$

where $\mathcal{A}^{*}, \mathcal{B}^{*}$ are the duals of the operators $\mathcal{A}, \mathcal{B}$ respectively (see equation 3) with $F, G$ satisfying the assumptions of Theorem 4. Then, for every $\nu_{0} \epsilon$ $\Pi_{\text {rba }}(H)$, equation (7) has at least one weak solution $\nu \in L_{\infty}^{w}\left(I, \Pi_{r b a}\left(H^{+}\right)\right) \subset$ $L_{\infty}^{w}\left(I, \Sigma_{r b a}\left(H^{+}\right)\right)$in the sense that for each $\phi \in D(\mathcal{A}) \cap \mathcal{D}(\mathcal{B})$ and $t \in I$, the following equality holds

$$
\nu_{t}(\phi)=\nu_{0}(\phi)+\int_{0}^{t} \nu_{s}(\mathcal{A} \phi) \pi(d s)+\int_{0}^{t} \nu_{s}(\mathcal{B} \phi) d s
$$

Proof. Proof will appear in [6].

REMARK 7 Note that Corollary 6 proves existence of (measure) solutions of Kolmogorov equation (7) with unbounded coefficients generalizing similar results of [7] for parabolic and elliptic equations on finite dimensional spaces.

The following corollary asserts uniqueness.

COROLlary 8 (UnIQUEness) Suppose the assumptions of Corollary 6 hold. Then the solution (weak solution) of the evolution equation (7) is unique.

Proof. Proof will appear in [6].

REMARK 9 Using this result we can prove the uniqueness of mild and hence weak solution of the stochastic measure equation (6). 


\section{MEASURABLE VECTOR FIELDS}

In many applications, $F, G$ and $\Gamma$ may not be even continuous. However, assuming that they are bounded Borel measurable, it is possible to prove existence results similar to those of deterministic evolutions [5].

Consider the control system

$$
\begin{aligned}
& d x(t)=A x(t) d t+F(x(t)) d t+\Gamma(x(t)) u(t, x) d t+G(x(t-)) M(d t) \\
& x(0)=x_{0},
\end{aligned}
$$

where $\Gamma: H \longrightarrow \mathcal{L}(\Xi, H)$ is a bounded Borel measurable map with $\Xi$ being another separable Hilbert space and $u: I \times H \longrightarrow \Xi$ is any bounded Borel measurable function representing the control. Let $B M(I \times H, \Xi)$ denote the class of bounded Borel measurable functions from $I \times H$ to $\Xi$. Furnished with the uniform norm topology,

$$
\|u\| \equiv \sup \left\{|u(t, x)|_{\Xi},(t, x) \in I \times H\right\},
$$

it is a Banach space. We present here a result analogous to that of Theorem 4 with the major exception that in the present case the measure solutions are no longer regular. They are bounded finitely additive measure valued processes.

Theorem 10 Consider the system (9). Suppose $\{A, M\}$ satisfy the assumptions of theorem 4, $F: H \longrightarrow H, G: H \longrightarrow \mathcal{L}(E, H)$ and $\Gamma: H \longrightarrow$ $\mathcal{L}(\Xi, H)$ are Borel measurable maps bounded on bounded sets. Then, for every $x_{0}$ for which $P\left\{\omega \in \Omega:\left|x_{0}\right|_{H}<\infty\right\}=1$, statistically independent of $M$, and $u \in B M(I \times H, \Xi)$, the evolution equation (9) has a unique measure solution

$$
\lambda^{0} \in M_{\infty, 2}^{w}\left(I \times \Omega, \Pi_{b a}\left(H^{+}\right)\right) .
$$

Proof. Proof will appear in [6].

\section{OPTIMAL FEEDBACK CONTROLS}

Consider the control system (9). For admissible controls, we use a weaker topology and introduce the following class of functions. Let $U$ be a closed bounded (possibly convex) subset of $\Xi$ and

$$
\mathcal{U} \equiv\{u \in B M(I \times H, \Xi): u(t, x) \in U \forall(t, x)\} .
$$

On $B M(I \times H, \Xi)$, we introduce the topology of weak convergence in $\Xi$ uniformly on compact subsets of $I \times H$ and denote this topology by $\tau_{w u}$. In other words, a sequence $\left\{u_{n}\right\} \subset B M(I \times H, \Xi)$ is said to converge to $u_{0} \in B M(I \times H, \Xi)$ in the topology $\tau_{w u}$ if, for every $v \in \Xi$,

$$
\left(u_{n}(t, x), v\right) \Xi \longrightarrow\left(u_{0}(t, x), v\right) \Xi
$$


uniformly in $(t, x)$ on compact subsets of $I \times H$. We assume that $\mathcal{U}$ has been furnished with the relative $\tau_{w u}$ topology and $\mathcal{U}_{a d}$ any $\tau_{w u}$ sequentially compact (possibly) convex subset of $\mathcal{U}$ and choose this set for admissible controls.

We consider the Lagrange problem $P 1$ : Find $u^{o} \in \mathcal{U}_{a d}$ that minimizes the cost functional

$$
J(u) \equiv \mathcal{E} \int_{0}^{T} \ell(t, x(t)) d t,
$$

where $\ell$ is any real valued Borel measurable function on $I \times H$ which is bounded on bounded sets. Since, under the general assumptions of Theorem 4 and Theorem 10, the control system (9) has only measure solutions, the control problem as stated above must be reformulated in terms of measure solutions. For this purpose we introduce the operator $\mathcal{B}_{u}$ associated with the control $u$ as follows. Define, for $(t, \xi) \in I \times H$,

$$
\left(\mathcal{B}_{u} \phi\right)(t, \xi) \equiv\left(u(t, \xi), \Gamma^{*}(\xi) D \phi(\xi)\right) \Xi,
$$

where $\Gamma^{*}(\xi) \in \mathcal{L}(H, \Xi)$ is the adjoint of the operator $\Gamma(\xi)$. Clearly the operator $\mathcal{B}_{u}$ is well defined on $D(\mathcal{A}) \cap D(\mathcal{B})$. Then the correct formulation of the original control problem is given by $(P 1)$ : find $u^{o} \in \mathcal{U}_{a d}$ that minimizes the functional

$$
J(u) \equiv \mathcal{E} \int_{0}^{T} \int_{H} \ell(t, \xi) \lambda_{t}^{u}(d \xi) d t
$$

where $\lambda^{u}$ is the (weak) solution of equation

$$
\begin{aligned}
& d \lambda_{t}=\mathcal{A}^{*} \lambda_{t} \pi(d t)+\mathcal{B}^{*} \lambda_{t} d t+\mathcal{B}_{u}^{*} \lambda_{t} d t+<\mathcal{C}^{*} \lambda_{t-}, M(d t)>_{E}, \\
& \lambda_{0}=\delta_{x_{0}} .
\end{aligned}
$$

Note that the initial measure need not be a Dirac measure, it suffices if $\lambda_{0}=$ $\pi_{0} \in \Pi_{b a}(H)$.

For convenience of reference we identify this problem as $P_{1}$. We need the following result on continuous dependence of solutions on control.

LEMMA 11 Consider the system (12) with admissible controls $\mathcal{U}_{\text {ad }}$ as defined above, and suppose the assumptions of Theorem 10 hold and that $\Gamma: H \longrightarrow$ $\mathcal{L}(\Xi, H)$ is a bounded Borel measurable map. Then, for every $u \in \mathcal{U}_{\text {ad }}$, the system (12) has a unique weak solution $\lambda^{u} \in M_{\infty, 2}^{w}\left(I \times \Omega, \Pi_{b a}\left(H^{+}\right)\right)$and further, the control to solution map $u \longrightarrow \lambda^{u}$ from $\mathcal{U}_{a d}$ to $M_{\infty, 2}^{w}\left(I \times \Omega, \Sigma_{b a}\left(H^{+}\right)\right)$ is (sequentially) continuous with respect to the topologies $\tau_{w u}$ on $\mathcal{U}_{a d}$ and weak star topology on $M_{\infty, 2}^{w}\left(I \times \Omega, \Sigma_{b a}\left(H^{+}\right)\right)$.

Proof. Detailed proof will appear in [6].

Now we consider the control problem $P 1$. 
THEOREM 12 Consider the system (12) and the Lagrange problem (11) with admissible controls $\mathcal{U}_{\text {ad }}$. Suppose the assumptions of Lemma 11 hold and that $\ell$ is a Borel measurable real valued function defined on $I \times H$ and bounded on bounded sets and that there exists a function $\ell_{0} \in L_{1}(I)$ such that $\ell(t, \xi) \geq$ $\ell_{0}(t) \forall \xi \in H$. Then there exists an optimal control for the problem $P 1$.

Proof. Since $\ell$ is bounded from below by an integrable function $\ell_{0}$, we have $J(u)>-\infty, \forall u \in \mathcal{U}_{a d}$. Clearly if $J(u)=+\infty$ for all $u \in \mathcal{U}_{a d}$, there is nothing to prove. So suppose the contrary. Define $\inf \left\{J(u), u \in \mathcal{U}_{\text {ad }}\right\}=m$, and let $\left\{u^{n}\right\} \subset \mathcal{U}_{a d}$ be a minimizing sequence. Since $\mathcal{U}_{a d}$ is $\tau_{w u}$ sequentially compact, there exists a subsequence, relabeled as the original sequence, and a control $u^{o} \in \mathcal{U}_{a d}$ such that $u^{n} \stackrel{\tau_{w u}}{\longrightarrow} u^{o}$. Then by virtue of Lemma 11, along a further subsequence if necessary, we have $\lambda^{u^{n}} \stackrel{w^{*}}{\rightarrow} \lambda^{u^{o}}$. Note that the functional (11) is linear in $\lambda^{u}$ and bounded (since $\left\{u^{n}\right\}$ is a minimizing sequence) and hence continuous along the minimizing sequence $\left\{\lambda^{u^{n}}\right\}$. Thus $\lim _{n \rightarrow \infty} J\left(u^{n}\right)=J\left(u^{o}\right)=m$ and $u^{o}$ is the optimal control.

Next we consider the control problem $P 2$ :

$$
J(u) \equiv \mathcal{E} \int_{I \times H}\{\ell(t, \xi)+\rho(\xi)|u(t, \xi)| \Xi\} \lambda_{t}^{u}(d \xi) d t \longrightarrow \inf ,
$$

where $\rho$ is a nonnegative bounded Borel measurable function on $H$ with compact support and $\lambda^{u}$ is the weak solution of the stochastic evolution equation (12) corresponding to control $u$.

Theorem 13 Consider the Lagrange problem P2(13) subject to the dynamics of system (12) with admissible controls $\mathcal{U}_{\text {ad }}$. Suppose $\ell$ satisfies the conditions as in Theorem 12, and $\rho$ is any nonnegative bounded Borel measurable function on $H$ having compact support. Then there exists an optimal control for the problem $P 2$.

Proof. Again by virtue of the assumption on $\ell$, we have $J(u)>-\infty$. If $J(u) \equiv+\infty$ for all $u \in \mathcal{U}_{a d}$ there is nothing to prove. So we may assume the contrary. Let $\left\{u^{n}\right\}$ be a minimizing sequence so that

$$
\lim _{n \rightarrow \infty} J\left(u^{n}\right)=\inf \left\{J(u), u \in \mathcal{U}_{a d}\right\} \equiv \tilde{m} .
$$

We show that the second term of the objective functional (13), denoted by $J_{2}$, is $\tau_{w u}$ lower semi continuous on $\mathcal{U}_{a d}$. Since $\mathcal{U}_{a d}$ is $\tau_{w u}$ sequentially compact, the sequence $\left\{u^{n}\right\}$ contains a subsequence, relabeled as the original sequence, which converges in $\tau_{w u}$ topology to an element $u^{o} \in \mathcal{U}_{a d}$. Consider the value of $J_{2}$ at $u^{o}$,

$$
J_{2}\left(u^{o}\right) \equiv \mathcal{E} \int_{I \times H} \rho(\xi)\left|u^{o}(t, \xi)\right|_{\Xi} \lambda_{t}^{u^{o}}(d \xi) d t .
$$


Since $u^{o}(t, \xi)$ is a $\Xi$ valued bounded Borel measurable function, by HahnBanach theorem there exists a $B_{1}(\Xi)$ valued bounded measurable function $\eta^{\circ}$ on $I \times H$ such that

$$
\left|u^{o}(t, \xi)\right|_{\Xi}=\left(u^{o}(t, \xi), \eta^{o}(t, \xi)\right)_{\Xi}, \forall(t, \xi) \in I \times H .
$$

In fact one can take $\eta^{o}(t, \xi)=u^{o}(t, \xi) /\left|u^{o}(t, \xi)\right| \Xi$. Using Lemma 11 and some functional analytic arguments one can verify that

$$
J_{2}\left(u^{o}\right) \leq \liminf _{n \rightarrow \infty} J_{2}\left(u^{n}\right)
$$

Thus $J_{2}$ is $\tau_{w u}$ lower semicontinuous and it follows from continuity of the first term that $J$ is $\tau_{w u}$ lower semicontinuous. The existence now follows from $\tau_{w u}$ sequential compactness of $\mathcal{U}_{a d}$.

Another interesting control problem, identified as $P 3$, consists of maximizing the functional:

$$
\left.J(u)=f\left(\mathcal{E} \lambda_{t_{1}}^{u}\left(\varphi_{1}\right), \cdots, \mathcal{E} \lambda_{t_{d}}^{u}\left(\varphi_{d}\right)\right)\right\} \rightarrow \sup
$$

where $f: R^{d} \longrightarrow R$ is a function, and $\left\{\varphi_{i}\right\} \in B(H)$ is a finite set of bounded real valued Borel measurable functions on $H$.

THEOREM 14 Consider the system (12) with admissible controls $\mathcal{U}_{\text {ad }}$ and suppose the assumptions of Lemma 11 hold. Further, suppose the stochastic vector measure $M$ is nonatomic and the associated quadratic variation measure $\pi$ is absolutely continuous with respect to the Lebesgue measure and the function $f$ is upper semicontinuous from $R^{d}$ to $R$ and $\left\{\varphi_{i}\right\} \in B(H)$ are real valued bounded Borel measurable functions. Then the Problem P3 has a solution.

Proof. For proof see [6].•

A fourth interesting problem, identified as $P 4$, can be stated as follows. Let $\Psi \in B(H)$ and $g \in C_{b}(R)$ be given. The problem is to find a control that minimizes (maximizes) the functional

$$
J(u) \equiv \mathcal{E} g\left(\lambda_{T}^{u}(\Psi)\right)
$$

THEOREM 15 Consider the system (12) with admissible controls $\mathcal{U}_{\text {ad }}$ and suppose the assumptions of Lemma 11 hold. Further, suppose $\{M, \pi\}$ satisfy the assumptions of Theorem 14 and $g \in C_{b}(R)$ and $\Psi \in B(H)$. Then the Problem P4 has a solution.

Proof. Proof will appear in [6].

REMARK 16 For construction of optimal controls, it is essential to develop necessary and sufficient conditions for optimality. We leave this as an open problem for the readers. 
REMARK 17 In section 5, the set $\mathcal{U}_{a d}$ is assumed to be $\tau_{w u}$ sequentially compact. The author feels that simple $\tau_{w u}$ compactness is sufficient. In general topological spaces compactness and continuity can all be described in terms of nets. Lemma 11 can be proved using nets and since all the results following Lemma 11 depend only on $\tau_{w u}$ continuity and compactness, they remain valid under this relaxed condition.

\section{References}

[1] N.U. Ahmed. Measure Solutions for Semi-linear Evolution Equations with Polynomial Growth and Their Optimal Controls. Discussiones Mathematicae, Differential Inclusions., 17, 5-27, 1997.

[2] N.U. Ahmed. Measure Solutions for Semilinear Systems with Unbounded Nonlinearities. Nonlinear Analysis: Theory, Methods and Applications, 35, 487-503, 1999.

[3] N.U. Ahmed. Relaxed Solutions for Stochastic Evolution Equations on Hilbert Space with Polynomial Nonlinearities. Publicationes Mathematicae, Debrecen, 54 (1-2), 75$101,1999$.

[4] N.U. Ahmed. Measure Solutions for Evolution Equations with Discontinuous Vector Fields. Nonlinear Functional analysis and Applications. 9(3), 467-484, 2004.

[5] N.U. Ahmed. Measure Solutions for Impulsive Evolution Equations with Measurable Vector Fields. Journal of Math. Annal. and Appl, 2004 (submitted).

[6] N.U. Ahmed: Measure Valued Solutions for Stochastic Evolution Equations on Hilbert Space and Their Feedback Control. Discussiones Mathematicae, DICO, 2005, 25 (to appear).

[7] S. Cerrai. Elliptic and parabolic equations in $R^{n}$ with coefficients having polynomial growth. Preprints di Matematica, n.9, Scuola Normale Superiore, Pisa, 1995.

[8] G. Da Prato and J. Zabczyk. Stochastic Equations in Infinite Dimensions. Encyclopedia of Mathematics and its Applications, 44, Cambridge University Press, 1992.

[9] J. Diestel and J.J.Uhl,Jr.. Vecior Measures. Math. Surveys Monogr. 15, AMS, Providence, RI, 1977.

[10] N. Dunford and J.T. Schwartz. Linear Operators, Part 1. Interscience Publishers, Inc., New York, 1958.

[11] H. Fattorini. A Remark on Existence of solutions of Infinite Dimensional Non-compact Optimal Control Problems, SIAM J. Control and Optim. 35(4), 1422-1433, 1997.

[12] I. Gyongy and N.V. Krylov. On Stochastic Equations with Respect to Semimartingales, Stochastics no. 1, 1-21, 1980/81. 